this; (2) the need for regular communication; (3) enhanced mental health support.

\section{0-22 THE IMPACT OF A NEW CHILDREN'S PALLIATIVE CARE SERVICE ON PLACE OF DEATH: GIVING FAMILIES CHOICES}

1,2Joanna Elverson, ${ }^{1}$ Helen Aspey, ${ }^{2}$ Owen Lever, ${ }^{1}$ Ellie Bond ${ }^{1}$ Christine Mackerness, ${ }^{1}$ Rebekah Hoskins, ${ }^{1}$ Allison Shiell. ${ }^{1}$ Newcastle upon Tyne Hospitals NHS Trust, Newcastle upon Tyne, UK; ${ }^{2}$ St Oswald's Hospice, Newcastle upon Tyne, UK

\subsection{6/spcare-2021-Hospice.21}

Background Although the North East, North Cumbria region has an active paediatric palliative care network, until recently the region lacked a dedicated palliative care service covering all localities and settings.

Supporting choice in place of death is a core principle of palliative care (National Institute for Health and Care Excellence. End of life care for infants, children and young people, Guidance NG61, 2016, rev. 2019; National Palliative and End of Life Partnership, 2021; Siden, Miller, Straatman, et al., 2008; Wolff, Robert, Sommerer, et al., 2010), however, data for the region demonstrated that very few children with nonmalignant conditions received end of life care at home (Gibson-Smith , Jarvis, Fraser, online first 2020). In 2021 a new regional children's palliative care service was launched, funded by specialist commissioning. We report on the early impact of the service relating to place of death.

Aims and Methods The service aims to improve families' experience by providing choice in place of death through:

- Assisting local paediatric teams with advice and practical help.

- Supporting families with visits and telephone contact.

- Facilitating decision-making and advance care planning.

Robust evaluation has been built into the service from the outset including collection of family and professional feedback. Results In the first six months of the project, the service received over 50 referrals. The palliative care team supported 11 children with end-of-life care and $100 \%$ died in their preferred setting. Six children received end-of-life care at home and the team facilitated five rapid transfers. Feedback from professionals and families highlighted the impact of the new service on enabling end-of-life care in community settings. The service supported children from every part of the region working alongside teams from all the four hospices and many children's clinical teams in the region. Locality debriefs following a child's death consolidated learning and continue to build relationships with local partners.

Conclusion We present evidence that the new children's palliative care service has successfully enabled children to receive consistent end-of-life care across all settings in the region, thus facilitating greater choice for families.

\section{$0-23$ VIEWS AND EXPERIENCES OF YOUNG PEOPLE, PARENTS/CARERS AND PROFESSIONALS OF ADVANCE CARE PLANNING}

Ben Hughes', Katherine Knighting², Mary O'Brien², Anita Flynn². 'University of Bolton, Bolton, UK; ${ }^{2}$ Edge Hill University, Ormskirk, UK

10.1136/spcare-2021-Hospice.22
Background Advance care planning (ACP) for adults has been widely practised in the United States, Canada, Australia, and New Zealand and has been embedded in the United Kingdom (UK) since 2005 (Dixon \& Knapp, 2020; Stein \& Fineberg, 2013; Hughes, O’Brien, Flynn, et al., 2018; Hayhoe, Howe, Gillick, et al., 2011). Approximately 99,000 children and young people in the UK have a life-limiting or life-threatening condition and may benefit from ACP (Fraser, Gibson-Smith, Jarvis, et al., 2020). However, little is understood about engaging young people in their own ACP from the different perspectives of those involved in the process.

Aim(s) To explore the views and experiences of young people, their parents/carers and healthcare professionals in the advance care planning process. Objectives were to explore both optimal timing of ACP discussions and facilitators and barriers to engaging young people in their care planning.

Methods A qualitative multiple case study research design was used to recruit participants, with young people as the unit of analysis. Data were collected using semi-structured interviews and analysed using thematic analysis and critical discourse analysis.

Results Views and experiences were mixed across the case studies. ACP should be initiated when young people are in their mid-teens, their condition is stable, and prior to transition to adult care. Facilitators to engagement were reported to be age- and developmentally-appropriate communication, relationships developed prior to initiating ACP, and support for everyone in the process. Perceived hierarchies of power and potential for disjointed communication and relationships can lead to misperception of ACP, resulting in barriers to engaging young people and negative experiences of the process.

Conclusions Exploration and understanding of views and experiences of different, concurrent participant groups has relevance for policy and practice. Understanding optimal timing of ACP discussions and the pivotal roles of communication, relationships, and organisational structure and culture, can create facilitators or barriers to engaging young people, in their own care planning.

This study was completed as a $\mathrm{PhD}$, which was funded by Edge Hill University. There are no conflicts of interest.

\section{Poster Presentations}

\section{Inequalities of access}

\section{P-1 A POPULATION HEALTH APPROACH TO QUANTIFYING HEALTH INEQUALITIES IN HOSPICE CARE}

Emma Hodges. St Giles Hospice, West Midlands, UK

\subsection{6/spcare-2021-Hospice.23}

Quantifying key elements of hospice care at primary care network level enables more targeted and clear discussions within the emerging Integrated Care Systems. Population health management creates a prime opportunity for hospices, particularly combining specialist care with community development activities. The combination of these factors creates possibly one of the best opportunities for hospices to engage and tackle health inequalities relating to access to palliative care. 